\title{
THE ENIGMA OF CONSCIOUSNESS - PHILOSOPHIC AND SCIENTIFIC APPROACH
}

\author{
A Skutchanova A, Saniova B.
}

Comenius University in Bratislava, Jessenius Faculty of Medicine in Martin and University Hospital Martin, Clinic of Anaesthesiology and Intensive Medicine, Slovakia

\begin{abstract}
Consciousness is enigmatic beast. It is present with us, comforting, and intuitive core of us. Yet this familiar beast, if we try to touch and describe it, escapes.Questioning and exploring consciousness accompanies mankind since ever. In $20^{\text {th }}$ century we met never before seen states of severe disorders of consciousness - nonresponding people with eyes wide open. Also advancements in general anesthesia made us reexplore this phenomena.

Article sums up current philosophic and two most prominent current neuroscientific approaches to what consciousness is and how we can assess it in other human beings. These theories may extend our understanding of ourselves and also help to asses this core quality in our patients in moments, when they do not intelligibly respond or do not respond at all.
\end{abstract}

Key Words: Consciousness, Theories, Integration information theory, Neuronal Correlate of Consciousness

\section{INTRODUCTION}

\section{Touching the consciousness}

Consciousness is all we are and all we have: loose consciousness, and, as far as you are concerned, your own self and the entire world dissolve into nothingness [1]. We are identified with being conscious so strong, that, in normal situation, we have no problem to distinguish, who is conscious and who is not. We usually understand the inner perspective of consciousness and outer perspective on the rest as nonproblematic connection of two contrary perspectives - soul and body. This deeply rooted Cartesian (named by its author, $17^{\text {th }}$ century philosopher René Descartes) dualism threads western culture.It is so strong that it still makes some scientist to approach the sole or minimal unit which makes us conscious - the spirit mysteriously settled in machine (body) - Homunculus, the observer, single, nonmaterial entirety of non-physical nature watching the Cartesian theatre of consciousness $[2,3]$.

Thousand years of introspection and our actual inability to experience, how is it not to be conscious [4] - and explanations what will happen to us when body deceases powers our inherited concept, in which consciousness lives its own, body-independent life. This gap rises from contrast of either thousand years of introspective exploration of consciousness and neuroscience research in the other side. Consciousness appears different from outside and inside: from outside we can access oscillating neurons, which we describe in language of physics. From inside we experience our intimate world of thoughts and feelings [2]. We often think that we can reduce consciousness to alertness, because we are usually aware conscious what we pay attention to $[3,5,6]$. But, awareness is objective and physical, whereas consciousness is not. You can be vividly conscious while not aware of self or environment: take ketamine anesthesia with vivid dreams as an example [7].

Term "consciousness" is highly intuitive, vague, which brings uncertainty to philosophy, linguistics, but also neuroscience, psychologyand clinical medicine. The most common definition says that it is state, in which a human individual is capable of correct perception of

Address for correspondence:

Škutchanová Alena, MD, Clinic of Anesthesiology and Intensive Medicine, Comenius University, Jessenius Faculty of Medicine and University Hospital Martin, Kollarova Str. N.2, 036 59, Martin, Slovakia, Phone:+421 434203 683; e-mail: askutchanova@gmail.com 
oneself and environment and correctly react on outer stimuli. It is individual's inner signaling of himself and environment [8]. But this favorite definition seems problematic: you can be vividly conscious without verbal signalization, for example if you are flooded by external stimuli, your inner voice disappears. There are also prelingual children and people with global aphasia. The "correct" is in the eyes of observer, otherwise inaccessible.

Using dictionary definitions seems problematic: it meets semantic nuances of languages and different use of terms in phrases which supplement the term. If we ve been speaking in different language, we would perceive different world (Ludwig Witgenstein) [9]. Term consciousness itself exist in most of European languages for about 300 years, in some languages and cultures is absent until present [2]. Or, like in oriental languages, is a term usually used in similar context untranslatable directly. We will use this term and prism of western culture as fundamental for our paper.

To objectivize nature sciences Galilei introduced objective observer and split up body and mind [2].Natural sciences use empiric experiment. Its prerequisite is to isolate examined system and to manipulate inputs. This makes us able to maintain parameters almost constant and change just one or few of them. The advantage of high reductionismus reproducibility and objectivity. Its disadvantage is prerequisite that there is objective, from surveyed system independent observer.

Recent neuroscience made great approach to understand many mechanism of consciousness: the way brain respond on different stimuli, decision making, focus and many others. It is called easy problems of consciousness [10]. Most progress done in science of consciousness past years have been done in this field But still, scientific research methods are not well suited for studying the neuronal process that accompany our conscious experience [5]. This very experience, its subjective parts, is calledhard problem of consciousness. It consists of two elements, self and qualia [2,11]. Qualia are our direct, subjective, conscious experience [11], the phenomenal consciousness. Consciousness like ability to experience something, does not require sensimotor loops containing body and environment, neither demands self-reflection (or language) noris reducible to alertness. The other is self: term for grasping oneself, the one who experiences. Self always seems like unity, the mighty homunculus. But some neurological states and disorders indicate that it consists of numerous components, each of which could be assessed individually. Idea of unity then seems like illusion [6], but also modular point of view, in which we denude some of its aspects, insufficient (The whole is more than sum of its parts - Aristotle). Consciousness is divided from processes which create it. We do not have direct access to processed information (for example the ones behind arising emotions), we just experience them [12].

There is one more aspect of consciousness science, as notices von Hayek: Every apparatus has to be on higher degree of complexity than the one who it tries to explain. Principally we cannot understand brain. It is cybernetically too complicated. Brain - observer cannot span brain - object [11].

\section{Current approaches in consciousness}

Approaches to describe consciousness are as diverse and multiple as consciousness experience itself. You can sort it by disciplines, methods, whatever. You can even get lost in the flood of coming information, which are mostly in field of easy problems of consciousness. Then you peel down all the humbug of easy problems and their solutions, and except questioning what is and what is not consciousness, you find yourself left with question like the one posed by Francis Crick: "Where lies the moment of no consciousness and how we can evaluate in other being?"

Currently we have two main theories on problem of defining the essence or minimum needed for conscious experience.

Searching for what is: Neuronal correlate of consciousness.

One approach to answer the question of consciousness, "contents systematically corresponding to consciousness contents" or also revealing "substrates of conscious experience" 
[2] or minimal neuronal mechanisms sufficient enough in commonfor any conscious perception [13-15]. Sometimes it seems like posthumous child of phrenology, where every function has its place on the human skull - and all we did was just move inside.Beloved child of Aristotle and his scientific method (searching direct cause-and-effect mechanism that create consciousness) as described in Organon, definition of neural correlate of consciousness (NCC) forces term "minimal", because question in research is, which parts of brain are actually needed. Second method of research is to assess states of global deterioration like sleep, anesthesia or coma and ask, what changed in brain. Using electrophysiological methods we would search for gamma-band synchrony emitting areas $(30-70 \mathrm{~Hz}$ and more) $[16,17]$. Large-capacity models predict that gamma-synchrony emergence is based on reciprocal connectivity and thalamocortical loops [18-22]. Approximately 100ms after presentation of consciously perceived stimulus, -oscillations were noticed in large areas. This is interpreted as that transitional event of -synchrony converts parallel processes to common time frame. Or perhaps gamma-beta synchrony could enable integration activity between local areas, global theta-rhythm after this trigger could provide time frame for integration of more globally distributed patterns. If particular synchrony, for example gamma, exclusively correlates to NCC, is not clear. It is possible to record it in numerous brain areas, while some of them directly do not take part on conscious processes [23]. So likewise "consciousness", "correlate" is the other confused term [24].

Also it is not yet clear whether synchrony in the gamma range vanishes during early NREM sleep, during anesthesia, or even during seizures. Human studies are still inconsistent. It would seem that there can be synchrony without consciousness, though perhaps not consciousness without synchrony, at least in mammalian brains. It would be premature to conclude that synchrony in one or another frequency band is necessarily a maker of consciousness[23]. There is also enigma of hydraencephalia: children with virtually no cortex (except residues of cortex provided by anterior and posterior cerebral artery and malaticfalxcerebri). They can briefly interact with environment, have moods or discriminate people [25].

\section{Searching for what is not}

To "make (hard) science" we explore consciousness with empiric experiment. Its prerequisite is to isolate examined system and to manipulate inputs, which is easy done in experiment with external stimuli. That is the easy and most explored part. In classic research we usually asses changes in visual network (easy to be influences and anatomically and functionally best explored in primates) [6]. Other pathways of perception are less explored and even to smaller extent if they are not explicit, even when most information we get in everyday contact, is implicit. But consciousness can even exist without external input: with transcranial magnetic stimulation (TMS) during ketamine-induced unresponsiveness the result is awakefulness-like, complex spatiotemporal activation pattern. Subject report vivid dreams during period of behavioral unresponsiveness on external stimuli [7]. NCC also describes synchrony as framing many different activity patterns and multiple parallel processes into a single synchrony - consciousexperience. We can explore multiple NCCs for different sensory inputs [26].Particularly interesting is NCC for auditory stimuli, because of potential legal consequences in case of for example explicit awareness in general anesthesia.

That is explaining: until we meet ultimate, briefly occupying consciousness with no obvious internal structure: darkness, pure deep blue or brightsound. It would merely seem to require strong activation of relating neurons, no need of complex synchrony. Also, the idea of neuronal correlate given by active neurons bound in synchrony discounts the importance of the inactive ones: information specifying that particular unified experience must be conveyed by both active and inactive neurons. Yet for inactive neuron seems nothing to bind. On the other hand, for example in case of generalized seizures, most of cortical neurons are active synchronously - but this bind rather seem to vanish consciousness than make it more vivid [23]. 
Neuronal correlate of consciousness also calls for that switching point between consciousness and unconsciousness. We can never experience, how it is, to be unconscious. We can experience vanishing or emerging of consciousness, but not unconsciousness per se.

We also need methods to explore consciousness when no reports about phenomenal experience are available. As promising there is scanning extent of brain activation after transcranial magnetic stimulation $[7,27]$.

\section{Integrated Information Theory of Consciousness}

Classically, the reduction of uncertainty among number of alternatives constitutes information $[28,29]$. If you lie in bed and experience pure silence and darkness, one may thought, that it conveys little information. One should realize, however, that the informativness of what you experience lies not in how complicated it is to describe, but how many alternatives were ruled out when you experienced it. This is so simple that it was overlooked for long time.

It is just as essential to realize that the information associated occurrence of consciousness state is integrated information. Conscious experience is integrated as a whole - it cannot be subdivided into components. Since integrated information can only be generated within the complex and not outside its boundaries, it is necessarily subjective, private and related to a single point of view or perspective [30,31]. Theory suspects that given system as brain is likely to contain more than one complex, but there is only one main complex that integrates most information in given time. So the emergence of consciousness is not only spatial, but also temporal matter. If the complex is stimulated for lesser time that neurons need for perturbation, effect of the stimulation will not be manifested.

Functional disconnections may also lead to restriction of neural substrate of consciousness as seen in neurological neglect phenomena or possible in dreaming. As we have seen, studies show that early NREM sleep is associated either with breakdown of effective connectivity among cortical areas, and thereby loss of integration [32,33] or with a stereotypical global response suggestive of loss of repetitoire and thus information [32]. This reduction could be reason why consciousness is reduced or eliminated in absence seizures or other states characterized by hypersynchronous neuronal activity [23]

For mammalian corticothalamic system is characteristic that different parts of cortex are specialized for different function, yet a vast network of connections allow these parts to interact profusely. And the corticothalamic system is the one part of brain, which cannot be severely impaired without loss of consciousness. Computer simulations also show that units along multiple, segregated incoming or outgoing pathways are not incorporated within the repetitoire of the main complex. This may be why neural activity in different pathways (perhaps as far as V1), although triggering it, does not contribute directly to conscious experience. Nor does activity in efferent pathways (perhaps starting with motor primary cortex), though it is crucial for reporting each different experience[23]. While it is not easy to determine, at present, whether a particular group of neurons is excluded from main complex because of hard-wired anatomical constraints, or is transiently disconnected due to functional changes, set of elements underlying consciousness is not static, but form dynamic core or dynamic complex [32].

Integrated Information Theory goes beyond proposing a provisional list of candidate brain areas for the neural substrate of consciousness, and of seemingly important neural ingredients, such as synchronization, sustained or phasic firing or reentrant activity without a principled explanation of why they would be important or whether they would be always necessary. The integrated information theory also avoids the pitfalls associated with assigning conscious qualities to individual brain elements. In complex, both active and inactive neurons counts, just as the sound of piano is given both by the keys which are played and those which remain silent. The theory also predicts that consciousness depends exclusively on ability of a system to integrate information, whether or not has strong sense of self, 
language, emotion, or is immersed in an environment contrary to some common intuition. The theory also says that the presence and extent of consciousness can be determined, in principle, also in case in which we have no verbal report, such as infants or animals, or neurological conditions like disorders of consciousness, psychomotor seizures or catatonic states. The theory assign consciousness of systems, which can make choice between alternatives and thus it is not panpsychism[1]. The theory also implies that consciousness is not all-or-none property, but is graded [23]. We have this experience every night when falling asleep.

Integration Information Theory is in its infancy and lack the graces of fully developed theory. It is exceedingly difficult to compute amount of integration even for very small systems. It does not differentiate between flesh and silicon and attributes consciousness to other beings - but does not downplay the gulf between nervous system of worm and human.

There is other grace of this theory: the mathematical approach, quantification of consciousness by quantification the complexity of brain response on transcranial magnetic stimulation in patients with questionable behavioral response: there seems to be promising algorithm to asses this subgroup of patients and eventually reveal locked-in patients [27, 34]. Differently from for example low resolution brain tomography (LORETA), algorithm is not dependent on projecting onto predefined brain model, which can differ individually, and be significantly changed in brain damaged and rewired in healing process.

We can still ask unanswered question, when there is moment of no consciousness and how can we define it in other being.

\section{Conclusion}

\section{Why to have a theory on consciousness}

Consciousness is trivial, intelligible everyday matter, until we try to define it or until we meet borderline and disturbing states like vegetative state or try to explain effect of different anesthetics.

There is no accepted theory of consciousness, no principled theory that would tell us which systems, organic or artificial, are conscious and why [35]. We also have a detailed and very successful framework for matter and energy, but not for the body-mind problem [36]. As the consciousness is subjective, there is no direct way to monitor it in others. But for a start we have access to our own experiences, which can be used to formulate theories. We can also plausibly rely on indirect information, such as subjects description of their experiences [5]. There lies one of the traps: borderline states of unconsciousness are accompanied with aphasia and possibly amnesia.We can just try to interact with people not capable of verbal communication and asses their reaction on us and environment. Such methods have limitations, but they give us enough to get started. These theories and concepts will not be conclusively testable, so they will inevitably be more speculative than those of more conventional scientific disciplines.

The ultimate goal of a theory of consciousness is a simple and elegant set of fundamental laws, analogous to fundamental laws of physics. If we find a theory that fits the data better than any other theory, we will have good reason to accept it.

Until that we will meet again and again with consciousness diminishing and rising in our patients in general anesthesia and try to understand and catch the first signs of consciousness in patient emerging from coma. Or just wonder and ask.

We were born too late to explore the Earth, we were born too early to explore the universe, but we are born right in time to explore the consciousness.

\section{REFERENCES}

1. Tononi G. Consciousness as Integrated Information: a Provisional Manifesto. Biol. Bull. December 2008 vol. 215 no. 3 216-242

2. Gáliková S. Úvod do filozofie vedomia. Filozofická fakulta, Trnavská Univerzita, Trnava. 2009. ISBN 978-80-8082-286-6 
3. Sániová B. Intenzívna a resuscitačná starostlivosṫ o dospelého pacienta $z$ hladiska poškodenia nervového systému. Martin: Ján Farský - PROFA, 2009. 233 p. ISBN 978-80-969722-2-7

4. Scientific American Editors. The Secrets od Consciousness, Kindle edition. November 182013. ASIN: B00G8BQAJK

5. Chalmers DJ. Solving the "Hard Problem". Scientific American 286(4) 90-99 (April 2002)

6. Tononi G, Koch C. The Neual Corelates of Consciousnes. Ann. N.Y. Acad. Sci. 2008;1124:239 261.

7. Sarasso S, Boly M. Consciousness and Complexityduring UnresponsivenessInduced by Propofol, Xenon, and Ketamine. Volume 25, Issue 23, p3099-3105, 7 December 2015

8. Ambler Z. et al. Základy neurologie. Praha: Galén, 2011. 351 p ISBN 9788072627073

9. Doušek F. Hejno bez ptáků. Toito 2012. ISBN 978-80-260-3077-5

10. Chalmers DJ. Solving the "Hard Problem". Scientific American 286(4) 90-99 (April 2002)

11. Hulín I. Patofyziológia, siedme vydanie. Slovac Academic Press, 2009. 1073-1080. 1200p ISBN: 9788080950439

12. Ward LM. The thalamic dynamic core theory of conscious experience. Conscious Cogn. 2011;20(2):464-86. doi: 10.1016/j.concog.2011.01.007

13. Crick F, Koch C. Are we aware of neural activity in primary visual cortex? Nature 1995;375:121-123.

14. Crick F, Koch C. Consciousness and neuroscience. Cereb. Cortex 1998;8:97-107.

15. Crick F, Koch C. A framework for consciousness. Nat. Neurosci. 2003;6:119-126.

16. Crick F, Koch C. Some reflections on visual awareness. Cold Spring Harbor Symposia on Quantitative Biology 1990;55:953-962.

17. Llinas R, Ribary U, Contreras D, Pedroarena C. The neuronal basis for consciousness. Philos.Trans.R.Soc.Lond.B Biol. Sci. 1998;353:1841-1849.doi: 10.1098/rstb.1998.0336

18. Lumer ED, Edelman GM, Tononi G. Neural dynamics in amodel of the thalamocortical system. 2. The role of neural synchrony tested through perturbations of spike timing. Cerebral Cortex 1997;7:228-236.

19. Lumer ED, Edelman GM, Tononi G. Neural dynamics in a model of the thalamocortical system. 1. Layers, loops and the emergence of fast synchronous rhythms. Cerebral Cortex 1997;7:207-227.

20. Edelman GM. The Remembered Present: A Biological Theory of Consciousness. NewYork, NY: BasicBooks, 1989

21. Lamme VA. Towards a true neural stance on consciousness. TrendsCogn.Sci. 2006;10:494-501. doi: 10.1016/j.tics.2006.09.001

22. Melloni L, Molina C, Pena M, Torres D, Singer W, Rodriguez, E. Synchronization of neural activity across cortical areas correlates with conscious perception. J. Neurosci. 2007;27:2858-2865. doi:10.1523/JNEUROSCI.4623-06.2007

23. Laureys S, Tononi G et al. The neurology of consciousness: Cognitive neuroscience and neuropathology. Elsevier, 2009. ISBN 978-0-12-374168-4

24. deGraafa TA, Hsiehc P-J,Sacka AT. The 'correlates' in neural correlates of consciousness. Neuroscience \&Biobehavioral ReviewsVolume 36, Issue 1, January 2012, Pages 191-197

25. Merker B. Life expectancy in hydrencephaly. Clin Neurol Neurosurg. 2008;110(3):213-4. doi: 10.1016/j.clineuro.2007.11.011.

26. Chalmers DJ. Facing Up to the Problem of Consciousness. Journal of Consciousness Studies 2(3):200-19, 1995.

27. Ragazzoni A, Pirulli C, Veniero D et al. Vegetative versus minimally conscious states: a study using TMS-EEG, sensory and event-related potentials.PLoS One. 2013;8(2):e57069. doi: 10.1371/journal.pone.0057069. Epub 2013 Feb 27

28. Jones DS. Elementary Information Theory. New York: Oxford University Press, 1979

29. Cover TM, Thomas JA. Elemants of Information Theory, $2^{\text {nd }}$ edition. Hoboken, NJ: WileyInterscience 2006

30. Tononi G. An information integration theory of consciousness. BMC Neurosci 2004;5:42

31. Tononi G, Edelman GM. Consciousness, information integration, and the brain. Science 1998 282:1846-1851

32. Massimini M, Ferrarelli F, et al. Triggering sleep slow waves by transcranial magnetic stimulation. Proc Natl AcadSci USA 2007; 104:8496-8501

33. Massimini M, Ferrareli f. et al. Breakdown of cortical effective connectivity during sleep. Science 2005; 309:2228-2232 
34. Casali AG, Gosseries O, Rosanova M, Massimini M. A Theoretically Based Index of Consciousness Independent of Sensory Processing and Behavior. Science translational medicine 5(198): 198ral05 . August 2013

35. Koch C. Exploring the "Mind" of Bees. Scientific American Mind. 19(6), 18-19. (December 2008/January 2009)

36. Koch C. A “Complex” Theory. Scientific American Mind. 2009, 20(4): 1619.

Received: March,18,2016

Accepted: July,4,2016 